The University of San Francisco

USF Scholarship: a digital repository @ Gleeson Library |

Geschke Center

$12-30-2003$

\title{
Anomaly in Conformal Quantum Mechanics: From Molecular Physics to Black Holes
}

Horacio E. Camblong

University of San Francisco, camblong@usfca.edu

Follow this and additional works at: http://repository.usfca.edu/phys

Part of the Physics Commons

\section{Recommended Citation}

Camblong, Horacio E., "Anomaly in Conformal Quantum Mechanics: From Molecular Physics to Black Holes" (2003). Physics and Astronomy. Paper 6.

http://repository.usfca.edu/phys/6 


\title{
Anomaly in conformal quantum mechanics: From molecular physics to black holes
}

\author{
Horacio E. Camblong \\ Department of Physics, University of San Francisco, San Francisco, California 94117-1080, USA \\ Carlos R. Ordóñez \\ Department of Physics, University of Houston, Houston, Texas 77204-5506, USA \\ and World Laboratory Center for Pan-American Collaboration in Science and Technology, University of Houston Center, Houston, \\ Texas 77204-5506, USA \\ (Received 19 March 2003; revised manuscript received 18 August 2003; published 30 December 2003)
}

\begin{abstract}
A number of physical systems exhibit a particular form of asymptotic conformal invariance: within a particular domain of distances, they are characterized by a long-range conformal interaction (inverse square potential), the apparent absence of dimensional scales, and an $\mathrm{SO}(2,1)$ symmetry algebra. Examples from molecular physics to black holes are provided and discussed within a unified treatment. When such systems are physically realized in the appropriate strong-coupling regime, the occurrence of quantum symmetry breaking is possible. This anomaly is revealed by the failure of the symmetry generators to close the algebra in a manner shown to be independent of the renormalization procedure.
\end{abstract}

DOI: 10.1103/PhysRevD.68.125013 PACS number(s): 11.10.Gh, 03.65.Fd, 11.25.Hf, 11.30.Qc

\section{INTRODUCTION}

An anomaly is defined as the symmetry breaking of a classical invariance at the quantum level. This intriguing phenomenon has played a crucial role in theoretical physics since its discovery in the 1960s [1]. In addition to its use in particle phenomenology of the standard model [2] and its extensions, it has been a fruitful tool for the study of conformal invariance in string theory [3].

Surprisingly, the presence of an infinite number of degrees of freedom does not appear to be a prerequisite for the emergence of anomalies. This fact was first recognized within a model with conformal invariance: the two-dimensional contact interaction in quantum mechanics [4]. In conformal quantum mechanics, a physical system is classically invariant under the most general combination of the following time reparametrizations: time translations, generated by the Hamiltonian $H$; scale transformations, generated by the dilation operator $D \equiv t H-(\mathbf{p} \cdot \mathbf{r}+\mathbf{r} \cdot \mathbf{p}) / 4$; and translations of reciprocal time, generated by the special conformal operator $K \equiv 2 t D-t^{2} H+m r^{2} / 2$. These generators define the noncompact $\mathrm{SO}(2,1) \approx \mathrm{SL}(2, R)$ Lie algebra $[5]$

$$
[D, H]=-i \hbar H, \quad[K, H]=-2 i \hbar D, \quad[D, K]=i \hbar K .
$$

This symmetry algebra has also been recognized in the free nonrelativistic particle [6], the inverse square potential $[7,8]$, the magnetic monopole [9], the magnetic vortex [10], and various nonrelativistic quantum field theories $[6,11,12]$. Furthermore, conformal quantum mechanics has been fertile ground for the study of singular potentials and renormalization, using Hamiltonian [13-15] as well as path integral methods [16]. Most importantly, a variety of physical realizations of conformal quantum mechanics have been recently identified, as discussed in the next section.

The main goals of this paper are (i) to illustrate the relevance of conformal quantum mechanics for several physical problems, from molecular physics to black holes, and (ii) to show the details of the breakdown of the commutator algebra (1) for the long-range conformal interaction. In Sec. II we introduce a number of examples that can be regarded as physical realizations of conformal quantum mechanics. In Sec. III we show that the origin of the anomaly can be traced to the short-distance singular behavior of the conformal interaction. In Sec. IV we introduce a generic class of realspace regulators, within the philosophy of the effective-field theory program. In Sec. V we compute the anomaly for the regularized theory and show that it is independent of the details of the ultraviolet physics, and in Sec. VI we comment on various renormalization frameworks. After the conclusions in Sec. VII, we summarize a number of technical results: a derivation of the anisotropic generalization of the conformal long-range interaction (Appendix A); a study of interdimensional dependence (Appendix B); a derivation of the near-horizon properties of black holes (Appendix C); and a derivation of useful integral identities (Appendix D).

\section{RELEVANT PHYSICAL APPLICATIONS}

In recent years, diverse examples of systems have been studied from the viewpoint of the conformal algebra (1), assumed to be a representation of an approximate symmetry within specific scale domains. In the applicable conformally invariant domain, the relevant physics is described by a $d$-dimensional effective Hamiltonian

$$
H=\frac{p^{2}}{2 m}-\frac{g}{r^{2}},
$$

which involves a long-range conformal interaction; or, alternatively, by its anisotropic counterpart

$$
H=\frac{p^{2}}{2 m}-\frac{g}{r^{2}} F(\boldsymbol{\Omega}),
$$


where $\boldsymbol{\Omega}$ stands for the angular variables and $F(\boldsymbol{\Omega})$ is a generic anisotropy factor that accounts for the angular dependence. Equation (3) is discussed in Appendix A.

In the problems considered below, $\lambda=2 m g / \hbar^{2}$ is the dimensionless form of the coupling constant and $\nu=(d$ -2)/2; furthermore, the choice $\hbar=1=2 m$ will be made for the problem involving black holes. In all cases, the strongcoupling regime [14] is defined by the condition $g \geqslant g^{(*)}$, with a critical dimensionless coupling $\lambda^{(*)} \equiv \lambda_{l}^{(*)}=(l+\nu)^{2}$ (for angular momentum $l$ ), when the Hamiltonian model (2) is adopted [14]. In addition, in the strong-coupling regime, as deduced in Sec. III, an uncontrolled oscillatory behavior of the Bessel functions of imaginary order $i \Theta$ makes the conformal system singular and regularization is called for. The characteristic parameter $\Theta=\sqrt{\lambda-(l+\nu)^{2}}$ strictly corresponds to the Hamiltonian (2); in physical applications, such as those of Secs. II A, II B, and II C, we will define

$$
\Theta \equiv \Theta_{\mathrm{eff}}=\sqrt{\lambda_{\mathrm{eff}}-\lambda_{\mathrm{eff}}^{(*)}},
$$

which will turn out to be crucial in parametrizing the anomalous physics of the conformal system in the presence of symmetry breaking. In discussing these realizations, we will explicitly use a subscript to emphasize the effective nature of the parameter of Eq. (4) — as arising from a reduction framework. The same notational convention will apply to the dimensionality $\left(d_{\text {eff }}\right)$. As shown in Appendix B, even when interdimensional equivalences are introduced, the value of the parameter (4) is a dimensional invariant.

\section{A. Dipole-bound anions and anisotropic conformal interaction}

The three-dimensional $\left(d_{\text {eff }}=3\right.$ or $\left.\nu_{\text {eff }}=1 / 2\right)$ interaction between an electron (charge $Q=-e$ ) and a polar molecule (dipole moment p) was the first physical application to be recognized as a realization of this anomaly [17]. When the molecule is modeled as a point dipole, this interaction can be effectively described with an anisotropic long-range conformal interaction of the form (3): $V(\mathbf{r})=-g \cos \theta / r^{2}$, in which the polar angle $\theta$ is subtended from the direction of the dipole moment. For this potential, the dimensionless coupling is $\lambda=-2 m K_{e} p Q / \hbar^{2}=p / p_{0}$, with $m$ being the reduced mass of the system and $K_{e}$ the electrostatic constant. Thus, the relevant scale for phenomenological analyses is provided by $p_{0} \approx 1.271 D$ (where $D$ stands for the Debye).

As shown in Appendix A, in some sense, the generic anisotropic conformal interaction (3) - of which the electronmolecule interaction is a particular case - can be reduced to an effective isotropic conformal interaction for the zero angular-momentum channel [see Eq. (A7)]; this corresponds to an effective Hamiltonian of the type (2), with an appropriate effective coupling $\lambda_{\text {eff }}$. More precisely, this equivalence is achieved, after separation of variables in spherical coordinates, at the level of the radial equation. In addition, the corresponding value of $\lambda_{\text {eff }}$ is identical to the eigenvalue $\gamma$ of the angular equation, which is a function of the dipole coupling $\lambda$. The effective conformal parameter (4) becomes

$$
\Theta_{\mathrm{eff}}=\sqrt{\gamma-\nu_{\mathrm{eff}}^{2}},
$$

where $\lambda_{\text {eff }}^{(*)}=\nu_{\text {eff }}^{2}$ for each eigenvalue $\gamma$ of the angular equation. When this outline is implemented, according to the procedure of Ref. [17] or its generalization of Appendix A, the existence of a critical dipole moment $p^{(*)}$ for binding is predicted; the order of magnitude of its "conformal value," $\lambda^{(*)} \approx 1.279$, or $p^{(*)} \approx 1.625 \mathrm{D}$, has been verified in numerous experiments $[18,19]$. In particular, when binding occurs, extended states known as dipole-bound anions are formed. These conclusions have also been confirmed by detailed $a b$ initio calculations $[18,19]$ and by studies that incorporate the effects of rotational degrees of freedom [20], which also modify slightly the value of $p^{(*)}$.

In short, the central issue in this analysis-also shared by the other physical realizations discussed in this paper-is the existence of a conformally invariant domain whose ultraviolet boundary leads to the anomalous emergence of bound states via renormalization. As a result, these states break the original conformal symmetry of the model and modify the commutators (1), as we will show in the next few sections. This simple fact alone captures the essence of the observed critical dipole moment in polar molecules and leads to an analytical prediction for the energies of the conformal states, as discussed in Sec. VI and Appendix A.

\section{B. Near-horizon black hole physics}

A generic class of applications of conformal quantum mechanics arises from the near-horizon conformal invariance of black holes, its impact on their thermodynamics [21], and its extension to superconformal quantum mechanics [22]. In particular, analyses based on the Hamiltonian (2) have been used to explore horizon states $[23,24]$ and to shed light on black hole thermodynamics [24]. Another class of current applications [25] involves a many-body generalization of Eq. (2): the Calogero model, which has also been directly linked to black holes [26]. These remarkable connections seem to confirm the conjecture that it is the horizon itself that encodes the quantum properties of a black hole [27].

In this context, we consider the spherically symmetric Reissner-Nordström geometry in $D$ spacetime dimensions, whose metric

$$
d s^{2}=-f(r) d t^{2}+[f(r)]^{-1} d r^{2}+r^{2} d \Omega_{D-2}
$$

is minimally coupled to a scalar field $\Phi(x)$ with action ( $c$ $=1$ and $\hbar=1$ )

$$
S=-\frac{1}{2} \int d^{D} x \sqrt{-g}\left[g^{\mu \nu} \partial_{\mu} \Phi \partial_{\nu} \Phi+m^{2} \Phi^{2}\right] .
$$

In Eq. (6), $d \Omega_{D-2}$ stands for the metric on the unit ( $D$ -2)-sphere, $f(r)=1-2\left(a_{M} / r\right)^{D-3}+\left(b_{Q} / r\right)^{2(D-3)}$, and the lengths $a_{M}$ and $b_{Q}$ are determined from the mass $M$ and charge $Q$ of the black hole respectively [28]. In this approach, the conformal structure is revealed by a two-step procedure discussed in Appendix C and consisting of: (a) a reduction to an effective Schrödinger-like equation, to be analyzed in its frequency $(\omega)$ components; (b) the introduction of a near-horizon expansion in the variable $x=r-r_{+}$ [with $r=r_{ \pm}$being the roots of $f(r)=0$, and $r_{+} \geqslant r_{-}$]. Two 
distinct scenarios emerge from this reduction: the extremal and nonextremal cases, when $r_{+}=r_{-}$and $r_{+} \neq r_{-}$, respectively. We will omit any discussion of the extremal case, which is known to pose a number of conceptual difficulties and is otherwise beyond the scope of the framework presented in this paper. As for the nonextremal case, the following facts arise from this reduction:

(i) The ensuing effective problem is described by an interaction

$$
V(x)^{\text {(near horizon) }} \propto x^{-2},
$$

which is conformally invariant with respect to the nearhorizon coordinate $x$.

(ii) The effective Hamiltonian, still being a $d$-dimensional realization of the conformal interaction, does not have the usual form corresponding to the radial part of a multidimensional Schrödinger problem. In particular, the angular momentum variables appear at a higher order in the nearhorizon expansion.

(iii) The coupling constant $\lambda_{\text {eff }}$ is supercritical for all nonzero frequencies. This can be seen from Eq. (C11), which implies that

$$
\Theta_{\text {eff }}=\frac{\omega}{\left|f^{\prime}\left(r_{+}\right)\right|} .
$$

The conclusion from this procedure is that the relevant physics occurs in the strong-coupling regime, in which the framework discussed in this paper can be applied.

\section{Other applications}

While Secs. II A and II B conform to the title of this paper, applications in other areas of physics are also likely. Among these, the Efimov effect $[29,30]$ stands out. This effect is expected to arise in a three-body system with shortrange interactions, in which at least two of the two-body subsystems have virtual or bound $s$-states near zero energy. As in the case of the dipole-bound anions of Sec. II A, these are spatially extended and weakly bound states. Unfortunately, the combination of phenomenological parameters needed to form these states, together with their weakly bound nature, has defied experimental detection to date. Nonetheless, this effect is regarded as relevant in the description of the three-body nucleon interaction [31]. The most outstanding feature of these three-body interactions in three spatial dimensions is the fact that these problems are reduced to an effective equation with a long-range conformal interaction in the strong-coupling regime. In terms of possible experimental detection, this effect is currently being studied for the description of various systems, including helium trimers and nuclear three-body halos [30].

The conformal nature of the effective interaction, for the three-body systems described above, can be deduced as follows. Typically, one starts by introducing hyperspherical coordinates with hyperradius $\rho \equiv r$, in a $d_{\text {eff }}$-dimensional configuration space for the internal degrees of freedom; if the one-particle dynamics occurs in a $d$-dimensional space, then $d_{\text {eff }}=2 d$ for the internal dynamics of the three-body system (as the total number of coordinates is $3 d$, but $d$ of them are eliminated in favor of the center-of-mass coordinates). Consequently, when a hyperspherical adiabatic expansion [32] is combined with a Faddeev decomposition of the wave function [33], a reduction to a $d_{\text {eff }}=2 d$ realization of our conformal model (2) is obtained. These conclusions can be gleaned from the conformal nature of the effective adiabatic potentials $V_{\text {eff }}(r)$ arising from this reduction framework [30],

$$
V_{\text {eff }}(r)=-\frac{g_{\text {eff }}}{r^{2}}, \quad \lambda_{\text {eff }}=(d-1)^{2}+\Theta_{\text {eff }}^{2}, \quad d_{\text {eff }}=2 d,
$$

where $g_{\text {eff }}$ and $\lambda_{\text {eff }}$ are related as described after Eq. (3). Incidentally, due to the interdimensional equivalence of Appendix $\mathrm{B}$, this result is often quoted in its one-dimensional reduced form [from Eq. (B4)], $\lambda(d=1)=\lambda_{\text {eff }}-(d-1)^{2}$ $+1 / 4=\Theta_{\text {eff }}^{2}+1 / 4$. For example, for the all-important case of ordinary three-dimensional space, $d_{\text {eff }}=6$ and $\lambda_{\text {eff }}=4$ $+\Theta_{\text {eff }}^{2}$. Furthermore, the coupling constant in Eq. (10) depends upon the physical parameters defining the system: when the scattering lengths are large, it is function of the three ratios of particle masses. In particular, for the lowest angular eigenvalue of a three-body three-dimensional system of identical bosons with zero-range two-particle interactions, the characteristic conformal parameter (4) is approximately given by the solution of the transcendental equation [30]

$$
8 \sinh \left(\frac{\pi \Theta_{\mathrm{eff}}}{6}\right)=\sqrt{3} \Theta_{\mathrm{eff}} \cosh \left(\frac{\pi \Theta_{\mathrm{eff}}}{2}\right),
$$

so that $\Theta_{\text {eff }} \approx 1.006$, which corresponds to the strongcoupling regime.

In short, the essential feature shared by the problems discussed above is the existence of an effective description in terms of $\mathrm{SO}(2,1)$ conformal invariance, which results from a prescribed reduction framework. We now turn our attention to this generic effective problem, characterized by the Hamiltonian of Eq. (2). As different dimensionalities are required for the applications to which Eq. (2) refers, we will analyze this problem for the arbitrary $d$-dimensional case. Our goal is to investigate and characterize the possible realization of a conformal anomaly within this scope.

\section{CONFORMAL ANOMALY AND SHORT-DISTANCE PHYSICS}

Conformal symmetry is guaranteed at the quantum level when the naive scaling of operators, described by the algebra (1), is maintained. A measure of the deviation from this scaling is afforded by the "anomaly" [34]

$$
\begin{aligned}
\mathcal{A}(\mathbf{r}) & \equiv \frac{1}{i \hbar}[D, H]+H=\left[1+\frac{1}{2} \mathcal{E}_{\mathbf{r}}\right] V(\mathbf{r}) \\
& =\frac{r^{d-2}}{2} \nabla \cdot\left[\frac{\mathbf{r} V(\mathbf{r})}{r^{d-2}}\right]
\end{aligned}
$$


(valid for arbitrary $d$ spatial dimensions), in which $\mathbb{1}$ is the identity operator and $\mathcal{E}_{\mathbf{r}}=\mathbf{r} \cdot \boldsymbol{\nabla}$. At first sight, the right-hand side of Eq. (12) appears to be zero for any scale-invariant potential; however, upon closer examination, this apparent cancellation may break down at $r=0$, where the interaction is singular. Equations (12) and (13) can be directly applied to any of the interactions within the conformal quantum mechanics class, and reduce to the familiar results known for the two-dimensional contact interaction $[34,35]$. However, the most interesting case is provided by the Hamiltonian (2), whose symmetry breaking can be made apparent by means of the formal $d$-dimensional identity $\boldsymbol{\nabla} \cdot\left[\hat{\mathbf{r}} / r^{d-1}\right]$ $=\Omega_{d-1} \delta^{(d)}(\mathbf{r})$, in which $\Omega_{d-1}$ is the surface area of the unit $(d-1)$-sphere $S^{d-1}$; then,

$$
\mathcal{A}(\mathbf{r})=-g \frac{\Omega_{d-1}}{2} r^{d-2} \delta^{(d)}(\mathbf{r}) .
$$

Despite its misleading appearance, this term is not identically equal to zero, due to the singular nature of the interaction at $r=0$. The recognition of this remarkable singular term, as well as of its regularized and renormalized counterparts, leads to the central result of our paper: the proof of the existence of a conformal anomaly.

However, two important points should be clarified. First, Eq. (14) is merely a formal identity, whose physical meaning can only be manifested through appropriate integral expressions. Second, the coordinate singularity highlights the need to determine the behavior of the wave function near $r=0$. Therefore, nontrivial consequences of Eq. (14) can only be displayed by the expectation value with a normalized state $|\Psi\rangle$,

$$
\frac{d}{d t}\langle D\rangle_{\Psi}=\langle\mathcal{A}(\mathbf{r})\rangle_{\Psi}=-g \frac{\Omega_{d-1}}{2} \int d^{d} \mathbf{r} \delta^{(d)}(\mathbf{r})\left|r^{\nu} \Psi(\mathbf{r})\right|^{2} .
$$

A similar analysis applies to the anisotropic interaction of Eq. (3); in this case,

$$
\begin{aligned}
\frac{d}{d t}\langle D\rangle_{\Psi} & =\langle\mathcal{A}(\mathbf{r})\rangle_{\Psi} \\
& =-g \frac{\Omega_{d-1}}{2} \int d^{d} \mathbf{r} \delta^{(d)}(\mathbf{r})\left|r^{\nu} \Psi(\mathbf{r})\right|^{2} F(\mathbf{\Omega}) .
\end{aligned}
$$

It should be noticed that the intermediate steps leading to Eqs. (15) and (16) are based on formal identities involving the $d$-dimensional $\delta$ function. For the unregularized inverse square potential, the integrals in Eqs. (15) and (16) select the limit $r \rightarrow 0$ of the product $r^{\nu} \Psi(\mathbf{r})$, which is known to be proportional to a Bessel function of order $i \Theta$, with $\Theta$ defined in Eq. (4). This limit is ill defined in the strongcoupling regime, due to the uncontrolled oscillatory behavior of the Bessel functions of imaginary order. Consequently, a regularization procedure is called for; inter alia, this procedure will assign a meaningful value to Eqs. (15) and (16).

\section{REGULARIZATION AND RENORMALIZATION: THE EFFECTIVE-FIELD THEORY PROGRAM}

The Hamiltonian (2), in the strong-coupling regime, describes an effective system with singular behavior for shortdistance scales. This interpretation, in which regularization and renormalization are mandatory, is inspired by the effective-field theory program [36]. The required regularization procedure is implemented in real space, where the ultraviolet physics is replaced over length scales $r \leq a$. The effective theory that comes out of this renormalization is expected to be applicable within a domain of energies of magnitude $|E| \ll E_{a} \equiv \hbar^{2} / 2 m a^{2}$. The scale $E_{a}$ defines an approximate limit of the conformal regime from the ultraviolet side; effectively, this limit prevents the singular interaction from yielding unphysical divergent results for supercritical coupling.

Specifically, we consider a generic class of regularization schemes that explicitly modify the ultraviolet physics; each scheme is described by a potential $V^{(<)}(\mathbf{r})$, for $r \lessgtr a$, where $a$ is a small real-space regulator. An appropriate procedure for the selection of solutions of this singular conformal interaction was proposed in Ref. [37], using a constant potential for $r \leqq a$. Our approach is based on a generalization of this method, in which a core interaction $V^{(<)}(\mathbf{r})$ is introduced.

Incidentally, in this section, we consider a core $V^{(<)}(\mathbf{r})$ $\equiv V^{(<)}(r)$ with central symmetry $V^{(<)}(r)$. Even though this condition is not strictly necessary, it leads to a tractable derivation. Moreover, it is also consistent with the original rotational invariance of the isotropic singular interaction and captures the singular behavior of the potential, which originates from its radial dependence (even in the anisotropic case). The generalization for an anisotropic conformal interaction is nontrivial, but when this interaction is reduced to an effective radial problem, the procedure developed in this section can be applied.

The core interaction is subject to the conditions of finiteness

$$
-\infty<V_{0} \equiv \min \left[V^{(<)}(r)\right] \equiv-\frac{\hbar^{2}}{2 m} \frac{\aleph}{a^{2}}
$$

and continuous matching with the external inverse square potential at $r=a$,

$$
V^{(<)}(a)=V^{(>)}(a)=-g / a^{2} .
$$

It should be noticed that these restrictions imply that $V_{0}<0$ or $\aleph>0$, and that $\boldsymbol{\kappa}=\lambda+\varsigma$, where $\varsigma>0$ is the dimensionless energy difference between the minimum $V_{0}$ and the matching value (18). In addition, in this approach, the energies for the interior problem will be conveniently redefined from the minimum value $V_{0}$; specifically,

$$
U(r) \equiv V^{(<)}(r)-V_{0}, \quad \widetilde{E}=E-V_{0} .
$$

For the spherically symmetric long-range conformal interaction of Eq. (2), central symmetry leads to the separable solution 


$$
\Psi(\mathbf{r})=\frac{\check{Y}_{l \mathbf{m}}(\mathbf{\Omega}) v(r)}{r^{\nu}}, \quad \int d \Omega_{d-1}\left|\check{Y}_{l \mathbf{m}}(\boldsymbol{\Omega})\right|^{2}=\Omega_{d-1},
$$

in which $\check{Y}_{l \mathbf{m}}(\mathbf{\Omega})$ stands for the ultraspherical harmonics on $S^{d-1}$ [38], which have been conveniently redefined with a normalization integral equal to the solid angle $\Omega_{d-1}$. Then, the corresponding effective radial Schrödinger equation for $v(r)$ becomes

$$
\left\{\frac{d^{2}}{d r^{2}}+\frac{1}{r} \frac{d}{d r}+\left[k^{2}-\frac{(l+\nu)^{2}}{r^{2}}-\mathcal{V}(r)\right]\right\} v(r)=0,
$$

where $\mathcal{V}(r)=2 m V(r) / \hbar^{2}$ and $k^{2}=2 m E / \hbar^{2}$. In particular, for bound states, $k=i \kappa$ and Eq. (21) provides solutions of the form

$$
v_{a}(r)= \begin{cases}v^{(<)}(r)=B_{l, \nu} w_{l+\nu}(\widetilde{k} r ; \widetilde{k}) & \text { for } r \leqslant a, \\ v^{(>)}(r)=A_{l, \nu} K_{i \Theta}(\kappa r) & \text { for } r \geqslant a,\end{cases}
$$

in which $K_{i \Theta}(z)$ is the Macdonald function [39], and where $\widetilde{k}$ is defined from $\widetilde{E}=\hbar^{2} \widetilde{k}^{2} / 2 m$, so that $\widetilde{k}=\sqrt{-\kappa^{2}-\mathcal{V}_{0}}$, with $\mathcal{V}_{0}=2 m V_{0} / \hbar^{2}<0$. The regularizing core is arbitrary and $w_{l+\nu}(\tilde{k} r ; \tilde{k})$ is a particular real solution in that region,

$$
\left\{\frac{d^{2}}{d r^{2}}+\frac{1}{r} \frac{d}{d r}+\left[\widetilde{k}^{2}-\frac{(l+\nu)^{2}}{r^{2}}-\mathcal{U}(r)\right]\right\} w_{l+\nu}(\widetilde{k} r ; \widetilde{k})=0,
$$

where $\mathcal{U}(r)=\mathcal{V}(r)-\mathcal{V}_{0}$; as an example, $w_{l+\nu}(\widetilde{k} r ; \widetilde{k})$ is a Bessel function of order $l+\nu$ when the potential $V^{(<)}(\mathbf{r})$ is a constant.

The solution (22) can be completely determined by enforcing the following three additional physical conditions: (a) continuity at $r=a$ of the radial wave function; (b) continuity at $r=a$ of the logarithmic derivative of the radial wave function; and (c) normalization of the wave function. In what follows, these conditions will be stated using the auxiliary parameters

$$
\xi=\kappa a, \quad \widetilde{\xi}=\widetilde{k} a,
$$

which satisfy Eq. (19), i.e.,

$$
\widetilde{\xi}^{2}+\xi^{2}=\aleph
$$

Consequently, these conditions (a)-(c) become, respectively,

$$
\begin{aligned}
B_{l, \nu} w_{l+\nu}(\widetilde{\xi} ; \tilde{k}) & =A_{l, \nu} K_{i \Theta}(\xi), \\
\mathcal{L}_{l+\nu}^{(<)}(\widetilde{\xi} ; \tilde{k}) & =\mathcal{L}_{i \Theta}^{(>)}(\xi),
\end{aligned}
$$

and [cf. Eq. (20)]

$$
\int d^{d} \mathbf{r}|\Psi(\mathbf{r})|^{2}=\Omega_{d-1} \int_{0}^{\infty} d r r|v(r)|^{2}=1,
$$

where we have conveniently redefined the logarithmic derivatives from $\mathcal{L}_{i \Theta}^{(>)}(\xi) \equiv \mathcal{E}_{\xi}\left[\ln K_{i \Theta}(\xi)\right]$, with $\mathcal{E}_{\xi}=\xi \partial / \partial \xi$, and similarly for $\mathcal{L}_{l+\nu}^{(<)}(\widetilde{\xi} ; \widetilde{k})$ in terms of $w_{l+\nu}(\widetilde{\xi} ; \widetilde{k})$. Explicitly, Eq. (28) takes the form

$$
\Omega_{d-1}\left[B_{l, \nu}^{2} \widetilde{k}^{-2} \mathcal{J}_{l+\nu}(\widetilde{\xi} ; \widetilde{k})+A_{l, \nu}^{2} \kappa^{-2} \mathcal{K}_{i \Theta}(\xi)\right]=1,
$$

in which the normalization constants can be chosen to be real, and where

$$
\mathcal{K}_{i \Theta}(\xi) \equiv \int_{\xi}^{\infty} d z z\left[K_{i \Theta}(z)\right]^{2}
$$

and

$$
\mathcal{J}_{l+\nu}(\widetilde{\xi} ; \widetilde{k}) \equiv \int_{0}^{\widetilde{\xi}} d z z\left[w_{l+\nu}(z ; \widetilde{k})\right]^{2} .
$$

Equations (26) and (29) then provide the values of the constants $A_{l, \nu}$ and $B_{l, \nu}$; for example,

$$
B_{l, \nu}=\frac{\kappa}{\sqrt{\Omega_{d-1}}}\left\{\frac{\xi^{2}}{\widetilde{\xi}^{2}} \mathcal{J}_{l+\nu}(\widetilde{\xi} ; \widetilde{k})+\left[\frac{w_{l+\nu}(\widetilde{\xi} ; \widetilde{k})}{K_{i \Theta}(\xi)}\right]^{2} \mathcal{K}_{i \Theta}(\xi)\right\}^{-1 / 2} .
$$

For reasons that will become clear in the next section, it is convenient to rewrite Eqs. (30) and (31) in an alternative way, using the generalized Lommel integrals of Appendix D. First, the integral defined by Eq. (30), which applies to the external domain $(r \geqslant a)$, can be expressed as

$$
\mathcal{K}_{i \Theta}(\xi)=\frac{1}{2}\left[K_{i \Theta}(\xi)\right]^{2} \mathcal{M}_{i \Theta}^{(>)}(\xi),
$$

where

$$
\mathcal{M}_{i \Theta}^{(>)}(\xi) \equiv\left[\mathcal{L}_{i \Theta}^{(>)}(\xi)\right]^{2}+\Theta^{2}-\xi^{2} .
$$

Similarly, the integral defined by Eq. (31), which applies to the internal domain $(r \leqslant a)$, takes the form

$$
\mathcal{J}_{l+\nu}(\widetilde{\xi} ; \widetilde{k})=\frac{1}{2}\left[w_{l+\nu}(\widetilde{\xi} ; \widetilde{k})\right]^{2} \mathcal{M}_{l+\nu}^{(<)}(\widetilde{\xi} ; \widetilde{k})+\mathcal{U}_{l+\nu}(\widetilde{\xi} ; \widetilde{k}),
$$

where

$$
\mathcal{M}_{l+\nu}^{(<)}(\widetilde{\xi} ; \widetilde{k}) \equiv\left[\mathcal{L}_{l+\nu}^{(<)}(\widetilde{\xi} ; \widetilde{k})\right]^{2}+\left[\widetilde{\xi}^{2}-(l+\nu)^{2}-\widetilde{\xi}^{2} \check{U}(\widetilde{\xi} ; \widetilde{k})\right]
$$

and

$$
\mathcal{U}_{l+\nu}(\widetilde{\xi} ; \widetilde{k}) \equiv \int_{0}^{\widetilde{\xi}} d z z\left[w_{l+\nu}(z ; \widetilde{k})\right]^{2}\left[\left(1+\frac{1}{2} \mathcal{E}_{z}\right) \check{U}(z ; \widetilde{k})\right],
$$

with $\check{U} \equiv U / \widetilde{E}$ and $\mathcal{E}_{z}=z \partial / \partial z$. The Lommel integral relation (33) appears to be simpler than Eq. (35) because of the absence of an extra core $\breve{U}(z ; \widetilde{k})$ in the external domain. 
In addition, the continuity conditions of the potential, Eq. (18), and of the logarithmic derivatives, Eq. (27), imply the equality of the "matching functions" (34) and (36), i.e.,

$$
\mathcal{M}_{l+\nu}^{(<)}(\widetilde{\xi} ; \widetilde{k})=\mathcal{M}_{i \Theta}^{(>)}(\xi)
$$

As a corollary, a combined Lommel relation can be obtained by elimination of the matching functions from Eqs. (33) and (35),

$$
\mathcal{J}_{l+\nu}(\widetilde{\xi} ; \widetilde{k})-\mathcal{U}_{l+\nu}(\widetilde{\xi} ; \widetilde{k})=\left[\frac{w_{l+\nu}(\widetilde{\xi} ; \widetilde{k})}{K_{i \Theta}(\xi)}\right]^{2} \mathcal{K}_{i \Theta}(\xi)
$$

Even though the implementation of a renormalization procedure is a necessary condition for the emergence of the conformal anomaly, the actual details of this procedure are not explicitly required. It suffices to know that these details are to be consistently derived from Eqs. (24)-(39), which permit the exact evaluation of all relevant expectation values, and by enforcing the finiteness of a particular bound state energy.

\section{COMPUTATION OF THE CONFORMAL ANOMALY}

The value of the anomalous part of the commutator $[D, H]$ is given as the "anomaly" $\mathcal{A}(\mathbf{r})$ in Eq. (12). In Sec. III, this quantity was computed for the unregularized inverse square potential in terms of the formal identity (14); this expression, in turn, led to an ill-defined expectation value (15). This difficulty can be overcome when the singular conformal interaction is regularized according to the generic scheme introduced in Sec. IV. Then, Eq. (12) will in principle yield two different contributions: one for $r \leqslant a$ and one for $r \geqslant a$, with the latter being of the form (14); thus,

$$
\begin{aligned}
\mathcal{A}_{a}(\mathbf{r})= & {\left[\left(\mathbb{1}+\frac{1}{2} \mathcal{E}_{\mathbf{r}}\right) V^{(<)}(\mathbf{r})\right] \theta(a-r) } \\
& -g \frac{\Omega_{d-1}}{2} r^{d-2} \delta^{(d)}(\mathbf{r}) \theta(r-a),
\end{aligned}
$$

where $\theta(z)$ stands for the Heaviside function, is the regularized counterpart of Eq. (14). Explicitly, this leads to an expectation value

$$
\frac{d}{d t}\langle D\rangle_{\Psi}=\left[\left\langle\mathcal{A}_{a}(\mathbf{r})\right\rangle_{\Psi_{a}}^{(<)}+\left\langle\mathcal{A}_{a}(\mathbf{r})\right\rangle_{\Psi_{a}}^{(>)}\right]
$$

where the integration range is split into the two regions: 0 $\leqslant r \leqslant a$ and $r \geqslant a$. Moreover, the identically vanishing second term

$$
\left\langle\mathcal{A}_{a}(\mathbf{r})\right\rangle_{\Psi_{a}}^{(>)}=0
$$

in Eq. (40) shows that the source of the conformal anomaly is confined to an arbitrarily small region about the origin. This result can be confirmed from a straightforward replacement of Eq. (12) by $\mathcal{A}(\mathbf{r})=-\frac{1}{2}(d-2) V(\mathbf{r})+\frac{1}{2} \boldsymbol{\nabla} \cdot\{\mathbf{r} V(\mathbf{r})\}$, which is identically equal to zero for any domain that ex- cludes the origin, when applied to any homogeneous potential of degree -2 (a defining characteristic of the external conformal interaction).

Once Eq. (42) is established, the anomaly can be computed from the contribution arising from the ultraviolet domain $r \leqslant a$,

$$
\frac{d}{d t}\langle D\rangle_{\Psi}=\left\langle\mathcal{A}_{a}(\mathbf{r})\right\rangle_{\Psi_{a}}^{(<)}=\int_{r \leqslant a} d^{d} \mathbf{r}\left[\left(1+\frac{1}{2} \mathcal{E}_{\mathbf{r}}\right) V(r)\right]\left|\Psi_{a}(\mathbf{r})\right|^{2}
$$

In Eq. (43), $V(r) \equiv V^{(<)}(r)$ can be replaced using Eq. (19), and $\Psi_{a}(\mathbf{r})$ using Eqs. (20) and (22); when these substitutions are made and the dimensionless variable $\widetilde{\xi}$ in Eq. (24) is introduced, Eq. (43) becomes

$$
\begin{aligned}
\frac{d}{d t}\langle D\rangle_{\Psi}= & \frac{\Omega_{d-1} B_{l, \nu}^{2}}{\widetilde{k}^{2}} \int_{0}^{\tilde{\xi}} d z z\left[w_{l+\nu}(z ; \widetilde{k})\right]^{2} \\
& \times\left\{\left(1+\frac{1}{2} \mathcal{E}_{z}\right)\left[V_{0}+U\left(\frac{z}{\widetilde{k}}\right)\right]\right\} .
\end{aligned}
$$

Despite its cumbersome appearance, the integral in Eq. (44) can be easily evaluated once the definitions (31) and (37) are introduced, so that

$$
\begin{aligned}
\frac{1}{E} \frac{d}{d t}\langle D\rangle_{\Psi}= & \frac{1}{E} \frac{\Omega_{d-1} B_{l, \nu}^{2}}{\widetilde{k}^{2}}\left[V_{0} \mathcal{J}_{l+\nu}(\widetilde{\xi} ; \widetilde{k})+\widetilde{E} \mathcal{U}_{l+\nu}(\widetilde{\xi} ; \widetilde{k})\right] \\
= & \frac{\Omega_{d-1} B_{l, \nu}^{2}}{\kappa^{2}}\left\{\frac{\xi^{2}}{\widetilde{\xi}^{2}} \mathcal{J}_{l+\nu}(\widetilde{\xi} ; \widetilde{k})\right. \\
& \left.+\left[\mathcal{J}_{l+\nu}(\widetilde{\xi} ; \widetilde{k})-\mathcal{U}_{l+\nu}(\widetilde{\xi} ; \widetilde{k})\right]\right\}
\end{aligned}
$$

where $V_{0}$ was replaced through the relation (19) or (25), and $E=-\hbar^{2} \kappa^{2} / 2 m$. Furthermore, in Eq. (46), the difference $\mathcal{J}_{l+\nu}(\widetilde{\xi} ; \widetilde{k})-\mathcal{U}_{l+\nu}(\widetilde{\xi} ; \widetilde{k})$ can be evaluated employing Eq. (39), so that

$$
\begin{aligned}
\frac{1}{E} \frac{d}{d t}\langle D\rangle_{\Psi}= & \frac{\Omega_{d-1} B_{l, \nu}^{2}}{\kappa^{2}}\left\{\frac{\xi^{2}}{\widetilde{\xi}^{2}} \mathcal{J}_{l+\nu}(\widetilde{\xi} ; \widetilde{k})\right. \\
& \left.+\left[\frac{w_{l+\nu}(\widetilde{\xi} ; \widetilde{k})}{K_{i \Theta}(\xi)}\right]^{2} \mathcal{K}_{i \Theta}(\xi)\right\} .
\end{aligned}
$$

Finally, the coefficient $B_{l, \nu}$ can be eliminated using Eq. (32), which shows that the right-hand side of Eq. (47) is identically equal to one for any bound state. This remarkable simplification concludes the proof that the anomaly defined in Eq. (12) is indeed given by

$$
\frac{d}{d t}\langle D\rangle_{\Psi}=E
$$


where $E$ is the energy of the corresponding stationary normalized state.

In short, we have validated the relation (48) — which agrees with the formal prediction from properties of expectation values [34]. This validation has been established using a generic regularization procedure. Therefore, regardless of the renormalization framework used, an anomaly is generated. The generality of Eq. (48) makes it available for a variety of physical applications, and is a necessary condition when the theory is renormalized.

\section{RENORMALIZATION FRAMEWORKS}

In the previous section we showed that the property (48) and related symmetry-breaking results are independent of the details of the regularization procedure. Because of the generality of the real-space regularization approach presented in this paper, these results extend the two-dimensional analysis of Ref. [40] in a number of nontrivial ways:

(i) For arbitrary renormalization frameworks, other than the "intrinsic" one of Ref. [40] (see below).

(ii) For any dimensionality $d$. Again, the two-dimensional case of Ref. [40] has unique features that considerably simplify the derivation within the intrinsic framework. This is particularly relevant because the physical applications that appear to be most interesting are $d$-dimensional realizations of this phenomenon, with $d \equiv d_{\mathrm{eff}} \neq 2$.

(iii) For any bound state and angular momentum channel (and not just for the $l=0$ channel associated with the ground state considered in Refs. [34,35,40]).

In this section we highlight the relevance of these results with an overview of the real-space "effective," "intrinsic," and "core" renormalization frameworks (according to the presentation of Ref. [41]), and discuss their relationship to the present anomaly calculation. Despite their apparent differences, these frameworks share the basic physical requirement that the system is renormalized under the assumption that the ultraviolet physics dictates the possible existence of bound states of finite energy; the corresponding energies $E$ and values of $\kappa \propto \sqrt{|E|}$ are then required to remain finite.

In order to facilitate the implementation of this renormalization program, it is convenient to display the specific limiting form that Eq. (27) takes when $a \rightarrow 0$; more precisely,

$$
\cot [\alpha,(\Theta, \kappa a)]^{(\kappa a \ll 1)} \sim \frac{1}{\Theta} \mathcal{L}^{(<)}(\aleph),
$$

where $\mathcal{L}^{(<)}(\widetilde{k} a ; \widetilde{k}) \stackrel{(\kappa a \ll 1)}{\sim} \mathcal{L}^{(<)}(\aleph)$ and

$$
\alpha(\Theta, \kappa a) \equiv \Theta\left[\ln \left(\frac{\kappa a}{2}\right)+\gamma_{\Theta}\right],
$$

with $\gamma_{\Theta}=-\{$ phase $[\Gamma(1+i \Theta)]\} / \Theta$ (which reduces to the Euler-Mascheroni constant $\gamma$ [42] in the limit $\Theta \rightarrow 0$ ).

In the effective renormalization framework, the system is regularized maintaining finite values of $|E| \ll E_{a} \equiv \hbar^{2} / 2 m a^{2}$. This condition defines an asymptotic conformally invariant domain; within that domain, the condition $\kappa a \ll 1$ limits the ultraviolet applicability of this effective scheme. Most im- portantly, this condition is systematically applied to derive physical predictions in a direct manner, within the prescriptions of Sec. IV. As a result, Eq. (49) leads to the bound-state energy levels [41]

$$
E_{n}=E_{0} \exp \left(-\frac{2 \pi n}{\Theta}\right),
$$

in which $E_{0}<0$ is an arbitrary proportionality constant. This derivation also shows that, as ultraviolet physics sets in for $|E| \gtrsim E_{a}$ (that is, for $\kappa a \gtrsim 1$ ), no claim can be made as to the nature of the states on these deeper scales.

A few comments are in order regarding Eq. (51). First, it explicitly displays a breakdown of the conformal symmetry, by the introduction of a scale $\left|E_{0}\right|$ and an associated sequence of bound states. Second, the scale $\left|E_{0}\right|$ arises from the renormalization procedure. Third, as a renormalization scale, $\left|E_{0}\right|$ cannot be predicted by the conformal model and it is to be adjusted experimentally. Fourth, once the experimental determination is carried out, an unambiguous prediction [from Eqs. (48) and (51)] follows,

$$
\frac{E_{n+1}}{E_{n}}=\frac{\frac{d\langle D\rangle_{\Psi_{n+1}}}{d t}}{\frac{d\langle D\rangle_{\Psi_{n}}}{d t}}=\exp \left(-\frac{2 \pi}{\Theta}\right),
$$

within the range of applicability, $\kappa a \ll 1$. This is in agreement with the conclusions of phenomenological analyses of the Efimov effect [30].

The alternative intrinsic and core frameworks are characterized by the fact that the limit $\xi=\kappa a \rightarrow 0$ is strictly applied before drawing any conclusions about the physics. Therefore, in order to keep the bound-state energies and $\kappa$ values finite, a running coupling parameter is explicitly introduced, so that Eq. (49) is still maintained in this limit. The running parameter is either the conformal coupling $g$, in the intrinsic framework, or the strength $\aleph$ of the regularizing core interaction, in the core framework.

In the case of the intrinsic framework, the dependence $g$ $=g(a)$, equivalent to $\Theta=\Theta(a)$, is enforced. This leads to the asymptotic running behavior $\Theta \sim 0$, which ensures that the left-hand side of Eq. (49) remains well defined. This limiting procedure leads to the renormalization framework of Refs. [13,14]; in particular, Eq. (52) [with the condition $\Theta$ $\sim 0$ ] implies the existence of a single bound state. In its original form, the renormalization framework of Refs. $[13,14]$ was based upon a Dirichlet boundary condition, which we now reinterpret as an effective Dirichlet boundary condition $[16] u(r=a) \stackrel{(a \rightarrow 0)}{\sim} 0$, for the reduced radial wave function $u(r)=\sqrt{r} v(r)$. This result is guaranteed by the prefactor $\sqrt{r}$, regardless of the behavior of $v(r)$. As for $v(r)$, two distinct cases should be considered: (i) the special case characterized by the simultaneous assignments $d=2, l$ $=0$, and constant $V^{(<)}(\mathbf{r})$ [or, to be more precise, with $\mathrm{s}$

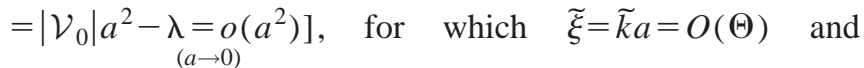
$\cos \alpha(\Theta, \kappa a) \sim 0$; (ii) the generic case, characterized by $d$ $\neq 2$, or $l \neq 0$, or $V^{(<)}(\mathbf{r})$ not being constant, for which the 
variable $\widetilde{\xi}=\widetilde{k} a$ acquires a nonvanishing limit value $\sqrt{\aleph}$ $=\sqrt{(l+\nu)^{2}+\varsigma}[$ as either $(l+\nu) \neq 0$ or $\varsigma \neq 0]$, and $\sin \alpha(\Theta, \kappa a) \stackrel{\sim}{\sim} 0$.

The smallness of the variable $\widetilde{\xi}$ for case (i) above is the main reason for the simplicity of the derivation of Ref. [40]. In effect, in this case, Eq. (43) can be approximated using the small-argument behavior of Bessel functions without explicitly computing full-fledged Lommel integrals. Thus, $\mathcal{J}_{l+\nu=0}(\tilde{k} a) \stackrel{(a \rightarrow 0)}{\sim} \Theta^{2} / 2 \quad$ and $\quad\left|B_{l=0, \nu=0}\right|$ $=\left|A_{l=0, \nu=0} K_{i \Theta}(\kappa a) / J_{0}(\tilde{k} a)\right|^{(a \rightarrow 0)} \sim /(\sqrt{\pi} \Theta)$, leading to $d\langle D\rangle_{\Psi} / d t \stackrel{(a \rightarrow 0)}{\sim} 2 \pi B_{0,0}^{2} \mathcal{J}_{0}(\tilde{k} a) V_{0} / \widetilde{k}^{2} \stackrel{(a \rightarrow 0)}{\sim} E$, as discussed in Ref. [40]. By contrast, for the generic case (ii), the analysis presented in this paper, based on the theory developed in Sec. IV and Appendix D, is inescapable.

Finally, in the core renormalization framework, the strength of the core interaction becomes a running coupling parameter: $\mathfrak{\aleph}=\mathfrak{\aleph}(a)$, but the conformal coupling $g$ remains constant $[15,43]$. As a result, Eq. (49) provides the limitcycle running that has been used in renormalization analyses of the three-body problem $[15,31,43]$.

Incidentally, the "effective" renormalization framework discussed in Ref. [41] (and summarized in this section) leads directly to a characterization of the thermodynamics of black holes. In essence, this amounts to a reinterpretation of 't Hooft's brick wall method [27], in which ultraviolet "new" physics sets in within a distance of the order of the Planck scale from the horizon. The computation of Appendix C shows that the leading behavior near the horizon is conformal and nontrivial, in that the effective system is placed in the supercritical regime. This asymptotic leading contribution, governed by the effective conformal interaction, requires renormalization and provides the correct thermodynamics [44]. It should be noticed that there is an alternative treatment, based upon the method of self-adjoint extensions, which has been recently discussed in Refs. [23,24].

\section{CONCLUSIONS}

Realizations of the conformal anomaly involve a breakdown of the associated $\mathrm{SO}(2,1)$ algebra. In this paper we have shown that the actual emergence and value of the conformal anomaly rely upon the application of a renormalization procedure, but are otherwise independent of the details of the ultraviolet physics. In this sense, the results derived herein are robust and totally general. As such, they are intended to shed light on the physics of any system with a conformally invariant domain for which the short-distance physics dictates the existence of bound states.

In particular, the dipole-bound anions of molecular physics and the Efimov effect are physical realizations of this unusual anomaly. In addition, the intriguing near-horizon physics of black holes appears to suggest yet another example of this ubiquitous phenomenon; the details of the thermodynamics arising from this conformal description will be reported elsewhere.

\section{ACKNOWLEDGMENTS}

This research was supported in part by NSF under Grant No. PHY-0308300 (H.E.C. and C.R.O.) and by the University of San Francisco Faculty Development Fund (H.E.C.). We also thank Dean Stanley Nel for generous travel support and Professors Cliff Burgess, Luis N. Epele, Huner Fanchiotti, and Carlos A. García Canal for early discussions of this work.

\section{APPENDIX A: ANISOTROPIC LONG-RANGE CONFORMAL INTERACTION AND CONFORMAL BEHAVIOR OF DIPOLE-BOUND ANIONS}

In this appendix we show the mathematical procedure that reduces the anisotropic inverse square potential to an effective isotropic interaction.

The Schrödinger equation for the Hamiltonian (3) can be separated in spherical coordinates by means of

$$
\Psi(\mathbf{r})=\frac{\Xi(\boldsymbol{\Omega}) u(r)}{r^{\nu+1 / 2}}
$$

with normalization

$$
\int d \Omega_{d-1}|\Xi(\boldsymbol{\Omega})|^{2}=1 .
$$

As a result, the angular part $\Xi(\boldsymbol{\Omega})$ of the wave function is no longer a solution to Laplace's equation on the unit $(d$ $-1)$-sphere $S^{d-1}$; instead, it satisfies the modified equation

$$
\hat{A} \Xi(\boldsymbol{\Omega})=\gamma \Xi(\boldsymbol{\Omega}),
$$

where

$$
\hat{A}=-\Lambda^{2}+\lambda F(\mathbf{\Omega})
$$

and $\Lambda^{2}=L^{2} / \hbar^{2}$ is the dimensionless squared angular momentum. The corresponding radial equation

$$
\frac{d^{2} u(r)}{d r^{2}}+\left(k^{2}+\frac{\gamma-\nu^{2}+1 / 4}{r^{2}}\right) u(r)=0
$$

is coupled to Eq. (A3) through the separation constant $\gamma$. Equation (A5) can be compared against the radial equation of an isotropic inverse square potential, which is obtained by another Liouville transformation [45] of the form (A1), but with ultraspherical harmonics instead of $\Xi(\boldsymbol{\Omega})$ and for $V_{\text {eff }}(\mathbf{r}) \propto r^{-2}$ without angular dependence; the effective equation

$$
\frac{d^{2} u(r)}{d r^{2}}+\left[k^{2}+\frac{\lambda_{\mathrm{eff}}-(l+\nu)^{2}+1 / 4}{r^{2}}\right] u(r)=0
$$

is identical to Eq. (A5) when the following identifications are made:

$$
V_{\mathrm{eff}}(\mathbf{r})=-\frac{g_{\text {eff }}}{r^{2}}, \quad g_{\text {eff }}=\frac{\hbar^{2}}{2 m} \lambda_{\text {eff }},\left.\quad \lambda_{\text {eff }}\right|_{l=0}=\gamma .
$$


Consequently, Eq. (A5) can be thought of as the radial part of a $d$-dimensional effective isotropic conformal interaction for $l=0$.

Furthermore, the values $\gamma$ are quantized from the angular equation (A3) and depend upon the coupling $\lambda$ of the anisotropic potential. This relationship can be made more explicit by expanding, in the ultraspherical-harmonic basis $Y_{l \mathbf{m}}(\boldsymbol{\Omega})$, the anisotropy factor

$$
F(\boldsymbol{\Omega})=\sum_{l, \mathbf{m}} F_{l \mathbf{m}} Y_{l \mathbf{m}}(\mathbf{\Omega})
$$

and the angular wave function

$$
\Xi(\boldsymbol{\Omega})=\sum_{l, \mathbf{m}} \Xi_{l \mathbf{m}} Y_{l \mathbf{m}}(\mathbf{\Omega}) .
$$

This decomposition yields the matrix counterpart of Eq. (A3), whence the anticipated relationship can be formally displayed by the infinite secular determinant

$$
D(\gamma, \lambda) \equiv \operatorname{det} M(\gamma, \lambda), \quad M(\gamma, \lambda)=-A(\lambda)+\gamma],
$$

in which 1 is the identity matrix; the matrix elements in Eq. (A10) are

$$
\begin{aligned}
\left\langle l \mathbf{m}|M(\gamma, \lambda)| l^{\prime} \mathbf{m}^{\prime}\right\rangle= & {[l(l+2 \nu)+\gamma] \delta_{l l^{\prime}} \delta_{\mathbf{m m}^{\prime}} } \\
& -\lambda \sum_{l^{\prime \prime}, \mathbf{m}^{\prime \prime}} I_{l \mathbf{m}, l^{\prime} \mathbf{m}^{\prime} ; l^{\prime \prime} \mathbf{m}^{\prime \prime}} F_{l^{\prime \prime} \mathbf{m}^{\prime \prime}},
\end{aligned}
$$

where

$$
I_{l \mathbf{m}, l^{\prime} \mathbf{m}^{\prime} ; l^{\prime \prime} \mathbf{m}^{\prime \prime}}=\int d \Omega_{d-1} Y_{l \mathbf{m}}^{*}(\boldsymbol{\Omega}) Y_{l^{\prime \prime} \mathbf{m}^{\prime \prime}}(\boldsymbol{\Omega}) Y_{l^{\prime} \mathbf{m}^{\prime}}(\boldsymbol{\Omega}) .
$$

Finally, the components $\Xi_{l \mathbf{m}}$ of the angular wave function can be formally obtained for every eigenvalue $\gamma$ in the usual way, and satisfy [from Eq. (A2)]

$$
\sum_{l, \mathbf{m}}\left|\Xi_{l \mathbf{m}}\right|^{2}=1 .
$$

As an example of this general theory, one can consider the particular three-dimensional case $(\nu=1 / 2)$ of the electronpolar molecule interaction described in Sec. II A. In this case, the matrix elements (A11) become

$$
\begin{aligned}
\langle\operatorname{lm}| M & (\gamma, \lambda)\left|l^{\prime} m^{\prime}\right\rangle \\
= & {[l(l+1)+\gamma] \delta_{l l^{\prime}} \delta_{m m^{\prime}} } \\
& -\lambda\left\{\sqrt{\frac{(l+m)(l-m)}{(2 l-1)(2 l+1)}} \delta_{l^{\prime}, l-1} \delta_{m m^{\prime}}\right. \\
& \left.+\sqrt{\frac{(l+m+1)(l-m+1)}{(2 l+1)(2 l+3)}} \delta_{l^{\prime}, l+1} \delta_{m m^{\prime}}\right\},
\end{aligned}
$$

which correspond to a matrix of block-diagonal form with respect to $m$ and tridiagonal in $l$. Then, the secular determinant (A10) factors out in the form $D(\gamma, \lambda)=\Pi_{m} D_{m}(\gamma, \lambda)$, with the reduced determinant $D_{m}(\gamma, \lambda)$ in the $m$ sector; thus, for given $m$, the equation $\operatorname{det} M(\gamma, \lambda)=0$ implies that

$$
D_{m}(\gamma, \lambda)=\left|\begin{array}{cccc}
\gamma & -\frac{\lambda}{\sqrt{3}} \sqrt{1-m^{2}} & 0 & \ldots \\
-\frac{\lambda}{\sqrt{3}} \sqrt{1-m^{2}} & (2+\gamma) & -\frac{\lambda}{\sqrt{15}} \sqrt{4-m^{2}} & \ldots \\
0 & -\frac{\lambda}{\sqrt{15}} \sqrt{4-m^{2}} & (6+\gamma) & \ldots \\
\ldots & \ldots & \ldots & \ldots
\end{array}\right|=0 .
$$

Equation (A15) has been used for the determination of the critical dipole moment $\lambda^{(*)} \approx 1.279$ [17] when $\gamma=\gamma^{(*)}$ $=1 / 4$. When the determinant is expanded (to high orders), additional roots appear for the critical condition $\gamma^{(*)}=1 / 4$ and for different values of $m$. This pattern also illustrates how one would completely solve the generic anisotropic problem: Eq. (A15) or its generalization (A10) can be used to obtain the eigenvalues $\gamma$ that correspond to a given coupling $\lambda$; these eigenvalues replace the usual angular momentum numbers. In the molecular physics case described above, the values of $\gamma$ can be easily evaluated numerically. When $\lambda<\lambda^{(*)}$, no such values produce binding; a first "binding eigenvalue" $\gamma_{0,0}$ is obtained when $\lambda \geqslant \lambda^{(*)}$, for the first root with $m=0$; as the strength $\lambda$ of the interaction increases, a second binding eigenvalue $\gamma_{0,1}$ is produced for the first root with $m=1$, when $\lambda \approx 7.58$ or $p \approx 9.63 \mathrm{D}$; the next eigenvalue $\gamma_{1,0}$ arises from the second root with $m=0$; etc. For each one of these values of $\gamma=\gamma_{j, m}$, an energy spectrum of conformal states is governed by Eq. (51), with $\Theta_{\text {eff }}$ given by Eq. (5). These bound states have been observed experimentally $[18,19]$ for the case when $\gamma_{0,0}$ is the only binding eigenvalue, 
a condition that corresponds to typical molecular dipole moments.

Most importantly, this analysis confirms that the conformal anisotropic problem can be reduced to the isotropic one, and the same symmetry-breaking considerations apply.

\section{APPENDIX B: DIMENSIONALITIES AND INTERDIMENSIONAL DEPENDENCE}

The spatial dimensionality $d_{\text {eff }}$ of a physical realization of conformal quantum mechanics is best characterized or defined as the dimension of the configuration space needed for a complete description of the dynamics within the conformal approximation. Typically, this quantity can be directly identified from the nature of the radial variable used in the description of scale and conformal symmetries.

For instance, with this convention, molecular anions can be naturally seen as a three-dimensional realization $\left(d_{\text {eff }}\right.$ $=3$ ); the Efimov effect, in a $d$-dimensional one-particle space, as a $(2 d)$-dimensional realization $\left(d_{\mathrm{eff}}=2 d\right)$; and the near-horizon conformal physics of black holes, in $D=d+1$ spacetime dimensions, as a $d$-dimensional realization $\left(d_{\text {eff }}\right.$ $=d)$.

Of course, there is a certain degree of arbitrariness in the selection of $d_{\text {eff }}$, due to the existence of a formal relationship connecting problems of different dimensionalities. This can be seen from the reduced Schrödinger-like radial equation of a conformal problem (2),

$$
\frac{d^{2} u(r)}{d r^{2}}+\left[k^{2}+\frac{\lambda-(l+\nu)^{2}+1 / 4}{r^{2}}\right] u(r)=0 .
$$

Equation (B1) depends on the number of spatial dimensions only through the combination $l+\nu$, a property known as interdimensional dependence [46]. As a consequence, the radial part of the solutions for any two conformal problems are identical when their coupling constants are related by

$$
\lambda\left(d^{\prime} ; l^{\prime}\right)=\lambda(d ; l)+\left(l^{\prime}-1+d^{\prime} / 2\right)^{2}-(l-1+d / 2)^{2} .
$$

Moreover,

$$
\Theta\left(d^{\prime}\right)=\Theta(d)
$$

is a dimensional invariant of these formal transformations. Correspondingly, the conformal physics is totally determined by the invariant value of this parameter.

However, the interdimensional equivalence of Eq. (B2) is severely limited by the fact that the full-fledged solutions (wave functions) are not identical, because the angular momenta are different in different dimensionalities. The only exception to this is the formal equivalence among the $l=0$ angular momentum channels of problems with arbitrary dimensionalities (as these channels do not involve additional dimension-dependent angular variables); in particular, an effective one-dimensional coupling can always be introduced for a $d$-dimensional problem with $l=0$ :

$$
\lambda\left(d^{\prime}=1 ; l^{\prime}=0\right)=\lambda(d ; l=0)+\frac{1}{4}-\frac{(d-2)^{2}}{4} .
$$

Even in the special case of the equivalence described by Eq. (B4), the full-fledged wave functions still retain a trace of the "physical dimensionality" $d$, because (with an obvious notation) $\left.u(r) \equiv \Psi\right|_{d=1}(r)=\left.r^{(d-1) / 2} \Psi\right|_{d}(r)$; for example, in the case of the three-dimensional Efimov effect, the fullfledged wave functions are of the form $\Psi(r) \propto r^{-5 / 2} u(r)$, reflecting the fact that $d_{\text {eff }}=6$.

The example of the near-horizon conformal behavior of black holes presents a number of peculiar features that deserve a separate treatment in Appendix C.

\section{APPENDIX C: NEAR-HORIZON CONFORMAL BEHAVIOR OF BLACK HOLES}

In this appendix we present an algebraic derivation of the conformal invariance exhibited near the horizon of a black hole.

From Eqs. (6) and (7), it follows that the equation of motion satisfied by the scalar field in the black-hole gravitational background is

$$
\begin{aligned}
\left(\square-m^{2}\right) \Phi \equiv & \frac{1}{\sqrt{-g}} \partial_{\mu}\left(\sqrt{-g} g^{\mu \nu} \partial_{\nu} \Phi\right)-m^{2} \Phi=-\frac{1}{f} \ddot{\Phi}+f \Phi^{\prime \prime} \\
& +\left(f^{\prime}+\frac{(D-2) f}{r}\right) \Phi^{\prime}+\frac{1}{r^{2}} \triangle_{D-2} \Phi-m^{2} \Phi \\
= & 0,
\end{aligned}
$$

where the dots stand for time derivatives and the primes for radial derivatives in the chosen coordinate description of the background, while $\triangle_{D-2}$ is the Laplacian on the unit ( $D$ $-2)$-sphere. In addition, by separation of the time and angular variables,

$$
\Phi(t, r, \boldsymbol{\Omega})=e^{-i \omega t} \varphi_{l \mathbf{m}}(r) Y_{l \mathbf{m}}(\mathbf{\Omega}),
$$

Eq. (C1) turns into

$$
\varphi^{\prime \prime}(r)+\left(\frac{f^{\prime}}{f}+\frac{(D-2)}{r}\right) \varphi^{\prime}(r)+\left(\frac{\omega^{2}}{f^{2}}-\frac{m^{2}}{f}-\frac{\alpha}{r^{2} f}\right) \varphi(r)=0,
$$

with $\alpha=l(l+D-3)$ being the eigenvalue of the operator $-\triangle_{D-2}$. Equation (C3) can be further reduced, by means of a Liouville transformation [45]

$$
\begin{aligned}
& \varphi(r)=g(r) u(r), \\
& g(r)=\exp \left\{-\frac{1}{2} \int\left[\frac{f^{\prime}}{f}+\frac{(D-2)}{r}\right] d r\right\}=f^{-1 / 2} r^{-(D-2) / 2},
\end{aligned}
$$

to its normal or canonical form

$$
u^{\prime \prime}(r)+I(r) u(r)=0
$$


with normal invariant

$$
\begin{aligned}
I(r)= & \frac{\omega^{2}}{f^{2}}-\frac{m^{2}}{f}-\left[\frac{(D-2)(D-4)}{4}+\frac{\alpha}{f}\right] \frac{1}{r^{2}} \\
& -\frac{1}{2} \frac{f^{\prime \prime}}{f}+\frac{1}{4} \frac{f^{\prime 2}}{f^{2}}-\frac{(D-2) f^{\prime}}{2 r f} .
\end{aligned}
$$

The conformal behavior of the Schrödinger-like equation (C5) near the horizon can be studied by means of an expansion in the variable

$$
x=r-r_{+},
$$

with $r=r_{+}$being the largest root of $f(r)=0$. The nonextremal case is characterized by the condition

$$
f_{+}^{\prime} \equiv f^{\prime}\left(r_{+}\right) \neq 0
$$

equivalent to $r_{+} \neq r_{-}$. Then,

$$
\begin{aligned}
& f(r)=f_{+}^{\prime} x[1+O(x)], \\
& f^{\prime}(r)=f_{+}^{\prime}[1+O(x)], \\
& f^{\prime \prime}(r)=f_{+}^{\prime \prime}[1+O(x)],
\end{aligned}
$$

where $f_{+}^{\prime \prime} \equiv f^{\prime \prime}\left(r_{+}\right)$. Thus, with corrective multiplicative factors of the order $[1+O(x)]$, it follows that $f^{\prime \prime} / f$ $\sim f_{+}^{\prime \prime} /\left(f_{+}^{\prime} x\right)$ and $f^{\prime} / f \sim 1 / x$, while $r \sim r_{+}$, so that the only leading terms in Eq. (C6) are $\omega^{2} / f^{2} \sim \omega^{2} /\left(f_{+}^{\prime} x\right)^{2}$ and $f^{\prime 2} /\left(4 f^{2}\right) \sim 1 /\left(4 x^{2}\right)$. As a result, Eq. (C5) is asymptotically reduced to the conformally invariant form

$$
u^{\prime \prime}(x)+\left[\frac{1}{4}+\frac{\omega^{2}}{\left(f_{+}^{\prime}\right)^{2}}\right] x^{-2}[1+O(x)] u(x)=0,
$$

where, by abuse of notation, we have replaced $u(r)$ by $u(x)$. Equation (C10) indicates the existence of an asymptotic conformal symmetry driven by the effective interaction

$$
\begin{aligned}
V_{\mathrm{eff}}(x) & =-\frac{\lambda_{\mathrm{eff}}}{x^{2}}, \\
\lambda_{\mathrm{eff}} & =\nu^{2}+\Theta_{\mathrm{eff}}^{2}, \quad \Theta_{\mathrm{eff}}^{2}=\left[\frac{\omega}{f^{\prime}\left(r_{+}\right)}\right]^{2},
\end{aligned}
$$

as follows by rewriting Eq. $(\mathrm{C} 10)$ in the $d$-dimensional format of Eq. (B1). This proves the claims made in Sec. II B and, in particular, Eqs. (8) and (9).

A final remark is in order. The effective Hamiltonian (C10) did not fall "automatically" within the $d$-dimensional format of Eq. (B1). The extra terms $\left.-\left[(l+\nu)^{2}-1 / 4\right)\right] / r^{2}$, usually obtained by reduction of a multidimensional Schrödinger equation in flat space, are still present, but at higher orders in the expansion with respect to the near-horizon coordinate $x$; in Eq. (C6), they correspond to

$$
\begin{aligned}
& -\left[\frac{(D-2)(D-4)}{4}+\frac{\alpha}{f}\right] \frac{1}{r^{2}} \\
& =-\left[\frac{(l+\nu)^{2}}{f}-\frac{1}{4}+\nu^{2}\left(1-\frac{1}{f}\right)\right] \frac{1}{r^{2}}=O\left(\frac{1}{x}\right)
\end{aligned}
$$

[with $\nu=(d-2) / 2=(D-3) / 2]$. Thus, the angular momentum-together with its associated dimensionality variable-decouples from the conformal interaction $(\mathrm{C} 11)$ in the near-horizon limit. It should be noticed that we had to rewrite Eq. $(\mathrm{C} 10)$ in the $l=0, d$-dimensional format in order to present this problem within our unified conformal model (2). Alternatively, one could write Eq. (C11) in a simpler one-dimensional reduced form [from Eq. (B4)], $\lambda(d=1)$ $=\lambda_{\text {eff }}-\nu^{2}+1 / 4=\Theta_{\text {eff }}^{2}+1 / 4$, with the same value for the dimensional invariant $\Theta_{\text {eff }}$.

\section{APPENDIX D: GENERALIZED LOMMEL INTEGRALS}

In this appendix we derive a generalization of the Lommel integrals [47] for an arbitrary Sturm-Liouville problem

$$
\begin{aligned}
\hat{L}_{x} v(x) & =\mu \varrho(x) v(x), \\
\hat{L}_{x} & =-\left\{\frac{d}{d x}\left[p(x) \frac{d}{d x}\right]+q(x)\right\},
\end{aligned}
$$

and apply it to the reduced radial Schrödinger equation (21). These generalized integrals are needed for the exact evaluation of expectation values in the anomaly calculation.

In what follows, we rewrite the differential equation (D1) in the form

$$
\frac{d}{d x}\left[p(x) v^{\prime}(x)\right]=-\left[\alpha^{2} \varrho(x)+q(x)\right] v(x),
$$

with an eigenvalue $\mu=\alpha^{2}$ and where the prime stands for a derivative with respect to $x$; moreover, $v(x)$ can be chosen to be a real function. Next, after conveniently multiplying both sides by $2 p(x) v^{\prime}(x)$, and integrating them with respect to $x$, Eq. (D3) turns into

$$
\begin{aligned}
& {\left.\left[p(x) v^{\prime}(x)\right]^{2}\right|_{x_{1}} ^{x_{2}}} \\
& \quad=-\int_{x_{1}}^{x_{2}} d x p(x)\left[\alpha^{2} \varrho(x)+q(x)\right] \frac{d}{d x}[v(x)]^{2},
\end{aligned}
$$

in which both the lower $\left(x_{1}\right)$ and upper limits $\left(x_{2}\right)$ are completely arbitrary. Finally, after integration by parts and rearrangement of terms, Eq. (D4) leads to 


$$
\begin{aligned}
\alpha^{2} \int_{x_{1}}^{x_{2}} d x[p(x) \varrho(x)]^{\prime}[v(x)]^{2} & \\
= & {\left.[v(x)]^{2}\left\{\left[p(x) \frac{v^{\prime}(x)}{v(x)}\right]^{2}+p(x)\left[\alpha^{2} \varrho(x)+q(x)\right]\right\}\right|_{x_{1}} ^{x_{2}} } \\
& -\int_{x_{1}}^{x_{2}} d x[p(x) q(x)]^{\prime}[v(x)]^{2},
\end{aligned}
$$

which generalizes the well-known second Lommel integral [47] of the theory of Bessel functions. A similar procedure could be applied for a generalization of the first Lommel integral, but this is not needed for the present purposes.

The integral relation (D5) can be rewritten in a convenient form for the reduced radial Schrödinger equation (21), which is of the generalized Bessel form

$$
\left\{\frac{d^{2}}{d x^{2}}+\frac{1}{x} \frac{d}{d x}+\left[\alpha^{2}-\mathcal{W}(x)\right]\right\} v(x)=0 .
$$

This is a particular case of the Sturm-Liouville equation (D3), with density function $\varrho(x)=x, p(x)=x$, and $q(x)$ $=-x \mathcal{W}(x)$; however, it is also true that a straightforward set of two Liouville transformations [45] makes Eqs. (D1) and (D6) formally equivalent to each other. For Eq. (D6), $[p(x) q(x)]^{\prime}=-\left[x^{2} \mathcal{W}(x)\right]^{\prime}$, and the final term in Eq. (D5) can be evaluated with the help of

$$
\frac{d}{d x}\left[\mathcal{W}(x) x^{2}\right]=2 x\left(1+\frac{1}{2} \mathcal{E}_{x}\right) \mathcal{W}(x)
$$

where 1 is the identity operator and $\mathcal{E}_{x}=x \partial / \partial x$, as in Sec. IV. As a consequence, Eq. (D5) becomes

$$
\begin{aligned}
\alpha^{2} \int_{x_{1}}^{x_{2}} d x x[v(x)]^{2} \\
=\left.\frac{1}{2}[v(x)]^{2}\left\{[\mathcal{L}(x)]^{2}+\left[(\alpha x)^{2}-x^{2} \mathcal{W}(x)\right]\right\}\right|_{x_{1}} ^{x_{2}} \\
\quad+\int_{x_{1}}^{x_{2}} d x x[v(x)]^{2}\left(1+\frac{1}{2} \mathcal{E}_{x}\right) \mathcal{W}(x),
\end{aligned}
$$

where $\mathcal{L}(x)=x v^{\prime}(x) / v(x)$ and both limits are still arbitrary. Equation (D8) is the desired generalization that can be directly applied to the reduced Schrödinger equations (21) and (23) to derive Eqs. (33) and (35), as we will show next.

First, for the interior problem $(r \leqslant a)$, Eq. (D8) turns into Eq. (35), by means of the substitutions

$$
\begin{aligned}
x & =r, \quad \alpha=\widetilde{k}, \quad x^{2} \mathcal{W}(x)=(l+\nu)^{2}+r^{2} \mathcal{U}(r), \\
v(x) & =w_{l+\nu}(\widetilde{k} r ; \widetilde{k}), \quad z=\widetilde{k} r,
\end{aligned}
$$

and with integration interval $z \in[0, \widetilde{\xi}]$, where $\widetilde{\xi}=\widetilde{k} a$. For this case, when $r^{2} \mathcal{U}(r) \rightarrow 0$, that is, for regular core potentials, the behavior of the differential equation at the origin implies that the contribution from the first term on the right-hand side of Eq. (D8) is zero for $r=0$.

Second, in a similar manner, for the exterior problem $(r$ $\geqslant a$ ), Eq. (D8) turns into Eq. (33), by means of the substitutions

$$
\begin{aligned}
x & =r, \quad \alpha=k=i \kappa, \quad x^{2} \mathcal{W}(x)=(l+\nu)^{2}-\lambda=-\Theta^{2}, \\
v(x) & =K_{i \Theta}(\kappa r), \quad z=\kappa r,
\end{aligned}
$$

and with integration interval $z \in[\xi, \infty]$, with $\xi=\kappa a$. Here, the behavior of the differential equation at infinity implies that the contribution from the first term on the right-hand side of Eq. (D8) is also zero at that point.
[1] S. B. Treiman, R. Jackiw, B. Zumino, and E. Witten, Current Algebras and Anomalies (World Scientific, Singapore, 1985).

[2] J. F. Donoghue, E. Golowich, and B. R. Holstein, Dynamics of the Standard Model (Cambridge University Press, Cambridge, England, 1992).

[3] M. B. Green, J. H. Schwartz, and E. Witten, Superstring Theory (Cambridge University Press, Cambridge, England, 1997), Vols. I and II.

[4] R. Jackiw, in M. A. B. Bég Memorial Volume, edited by A. Ali and P. Hoodbhoy (World Scientific, Singapore, 1991).

[5] B. Wybourne, Classical Groups for Physicists (Wiley, New York, 1974).

[6] U. Niederer, Helv. Phys. Acta 45, 802 (1972); C.R. Hagen, Phys. Rev. D 5, 377 (1972).

[7] R. Jackiw, Phys. Today 25(1), 23 (1972).

[8] V. de Alfaro, S. Fubini, and G. Furlan, Nuovo Cimento Soc. Ital. Fis., A 34, 569 (1976).

[9] R. Jackiw, Ann. Phys. (N.Y.) 129, 183 (1980).

[10] R. Jackiw, Ann. Phys. (N.Y.) 201, 83 (1990).

[11] R. Jackiw and S.Y. Pi, Phys. Rev. D 42, 3500 (1990).
[12] O. Bergman, Phys. Rev. D 46, 5474 (1992); O. Bergman and G. Lozano, Ann. Phys. (N.Y.) 229, 416 (1994).

[13] K.S. Gupta and S.G. Rajeev, Phys. Rev. D 48, 5940 (1993).

[14] H.E. Camblong, L.N. Epele, H. Fanchiotti, and C.A. García Canal, Phys. Rev. Lett. 85, 1590 (2000); Ann. Phys. (N.Y.) 287, 14 (2001); 287, 57 (2001).

[15] S.R. Beane, P.F. Bedaque, L. Childress, A. Kryjevski, J. McGuire, and U. van Kolck, Phys. Rev. A 64, 042103 (2001).

[16] H.E. Camblong and C.R. Ordóñez, Mod. Phys. Lett. A 17, 817 (2002); Phys. Rev. A 65, 052123 (2002); hep-th/0109003.

[17] H.E. Camblong, L.N. Epele, H. Fanchiotti, and C.A. García Canal, Phys. Rev. Lett. 87, 220402 (2001).

[18] R.D. Mead, K.R. Lykke, and W.C. Lineberger, J. Chem. Phys. 81, 4883 (1984); K.R. Lykke, D.M. Neumark, T. Andersen, V.J. Trappa, and W.C. Lineberger, ibid. 87, 6842 (1987); A.S. Mullin, K.K. Murray, C.P. Schulz, and W.C. Lineberger, J. Phys. Chem. 97, 10281 (1993).

[19] C. Desfrançois, H. Abdoul-Carime, N. Khelifa, and J.P. Schermann, Phys. Rev. Lett. 73, 2436 (1994), and references therein. 
[20] W.R. Garrett, Phys. Rev. A 3, 961 (1971); 22, 1769 (1980); D.C. Clary, J. Phys. Chem. 92, 3173 (1988); H. AbdoulCarime and C. Desfrançois, Eur. Phys. J. D 2, 149 (1998).

[21] S. Carlip, Phys. Rev. Lett. 82, 2828 (1999); Class. Quantum Grav. 16, 3327 (1999); Phys. Rev. Lett. 88, 241301 (2002); S.N. Solodukhin, Phys. Lett. B 454, 213 (1999).

[22] P. Claus, M. Derix, R. Kallosh, J. Kumar, P.K. Townsend, and A. Van Proeyen, Phys. Rev. Lett. 81, 4553 (1998); J.A. de Azcárraga, J.M. Izquierdo, J.C. Pérez Bueno, and P.K. Townsend, Phys. Rev. D 59, 084015 (1999); G. Papadopoulos, Class. Quantum Grav. 17, 3715 (2000); and references therein.

[23] T.R. Govindarajan, V. Suneeta, and S. Vaidya, Nucl. Phys. B583, 291 (2000).

[24] D. Birmingham, K.S. Gupta, and S. Sen, Phys. Lett. B 505, 191 (2001); K.S. Gupta and S. Sen, ibid. 526, 121 (2002).

[25] B. Basu-Mallick and K.S. Gupta, Phys. Lett. A 292, 36 (2001); B. Basu-Mallick, P.K. Ghosh, and K.S. Gupta, Nucl. Phys. B659, 437 (2003); Phys. Lett. A 311, 87 (2003).

[26] G.W. Gibbons and P.K. Townsend, Phys. Lett. B 454, 187 (1999).

[27] G. 't Hooft, Nucl. Phys. B256, 727 (1985).

[28] R. Myers and M.J. Perry, Ann. Phys. (N.Y.) 172, 304 (1986).

[29] V. Efimov, Phys. Lett. 33B, 563 (1970); Sov. J. Nucl. Phys. 12, 589 (1971); Comments Nucl. Part. Phys. 19, 271 (1990).

[30] E. Nielsen, D.V. Fedorov, A.S. Jensen, and E. Garrido, Phys. Rep. 347, 373 (2001).

[31] P.F. Bedaque, H.-W. Hammer, and U. van Kolck, Phys. Rev. Lett. 82, 463 (1999); Nucl. Phys. A646, 444 (1999).
[32] J. Macek, J. Phys. B 1, 831 (1968).

[33] L.D. Faddeev, Sov. Phys. JETP 12, 1014 (1961).

[34] G.N.J. Añaños, H.E. Camblong, C. Gorrichátegui, E. Hernández, and C.R. Ordóñez, Phys. Rev. D 67, 045018 (2003).

[35] J.G. Esteve, Phys. Rev. D 66, 125013 (2002).

[36] K.G. Wilson, Rev. Mod. Phys. 47, 773 (1975); J. Polchinski, Nucl. Phys. B231, 269 (1984); hep-th/9210046.

[37] L. D. Landau and E. M. Lifshitz, Quantum Mechanics, 3rd ed. (Pergamon, Oxford, 1977), pp. 114-117.

[38] Higher Transcendental Functions, edited by A. Erdélyi and the staff of the Bateman Manuscript Project (McGraw-Hill, New York, 1953), Vol. II, Chap. XI.

[39] Reference [38], Vol. II, Chap. VII.

[40] G.N.J. Añaños, H.E. Camblong, and C.R. Ordóñez, Phys. Rev. D 68, 025006 (2003).

[41] H.E. Camblong and C.R. Ordóñez, hep-th/0305035.

[42] Reference [38], Vol. I, Chap. I.

[43] M. Bawin and S.A. Coon, Phys. Rev. A 67, 042712 (2003).

[44] The black-hole entropy in $d$ dimensions is proportional to the "area" of the outer-horizon hypersurface: Ref. [28].

[45] A. R. Forsyth, A Treatise on Differential Equations, 6th ed. (Macmillan, London, 1929), Secs. 58-60.

[46] J. H. Van Vleck, in Wave Mechanics, the First Fifty Years, edited by W. C. Price et al. (Butterworth, London, 1973), p. 26.

[47] G. N. Watson, A Treatise on the Theory of Bessel Functions, 2nd ed. (Cambridge University Press, Cambridge, England, 1944), Sec. 5.11 . 